Document downloaded from:

http://hdl.handle.net/10251/119992

This paper must be cited as:

Lebtahi Ep-Kadi-Hahifi, L.; Romero Martínez, JO; Thome, N. (2018). Further results on generalized centro-invertible matrices. Numerical Algorithms. 80(4):1309-1328. https://doi.org/10.1007/s11075-018-0528-9

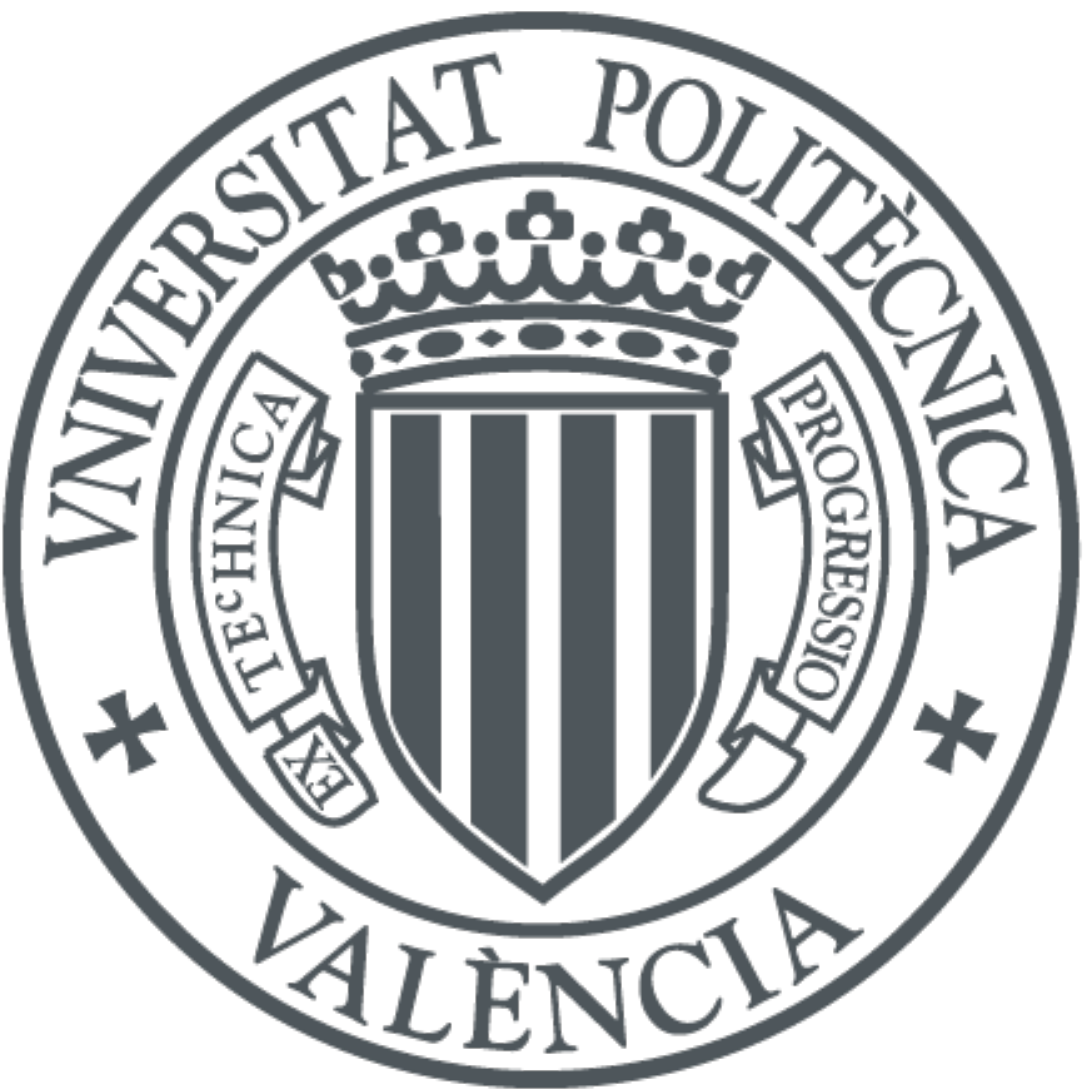

The final publication is available at

https://doi.org/10.1007/s11075-018-0528-9

Copyright Springer-Verlag

Additional Information 


\title{
Further results on generalized centro-invertible matrices
}

\author{
Leila Lebtahi* Óscar Romero ${ }^{\dagger} \quad$ Néstor Thome
}

\begin{abstract}
This paper deals with generalized centro-invertible matrices introduced by the authors in [Generalized centro-invertible matrices with applications, Applied Mathematics Letters, 38, 106-109, 2014]. As a first result, we state the coordinability between the classes of involutory matrices, generalized centro-invertible matrices, and $\{K\}$-centrosymmetric matrices. Then, some characterizations of generalized centro-invertible matrices are obtained. A spectral study of generalized centro-invertible matrices is given. In addition, we prove that the sign of a generalized centro-invertible matrix is $\{K\}$-centrosymmetric and that the class of generalized centro-invertible matrices is closed under the matrix sign function. Finally, some algorithms have been developed for the construction of generalized centro-invertible matrices.
\end{abstract}

AMS Classification: 15A09, 15A18

Keywords: Centro-symmetric matrices; centro-invertible matrices; spectral analysis; inverse problem; matrix sign function.

\section{Introduction}

Centrosymmetric matrices are those complex matrices $A \in \mathbb{C}^{n \times n}$ such that $A J_{n}=J_{n} A$ where $J_{n} \in \mathbb{C}^{n \times n}$ is the matrix with 1's on the secondary diagonal and 0's otherwise. An important property of centrosymmetric matrices is that if $A$ is a centrosymmetric matrix with $\gamma$ linearly independent eigenvectors, then $\gamma$ linearly independent eigenvectors of $A$ can be chosen to be symmetric or skewcentrosymmetric. The particular case of matrices that commute with a permutation matrix was studied in [13] by Stuart and Weaver. This class of matrices has been widely studied by considering their applications $[4,14,18]$. In [1], AbuJeib used them to analyze some spectral properties of regular magic squares. After applying the Sinc collocation method to Sturm-Liouville problems, the resulting matrices are centrosymmetric. Eigenspectrum properties of symmetric centrosymmetric matrices presented in [6] are applied for solving a generalized eigensystem of smaller dimensions than the original ones. Moreover, algorithms for solving centrosymmetric linear systems of equations are presented in [5]. Not only direct problems have been solved by using centrosymmetric matrices, but also the inverse eigenproblem and its approximation have been considered by

\footnotetext{
*Universitat de València, 46100. Valencia, Spain. E-mail: leila.lebtahi@uv.es.

†Universitat Politècnica de València, 46022. Valencia, Spain. E-mail: oromero@dcom.upv.es.

${ }^{\ddagger}$ Instituto Universitario de Matemática Multidisciplinar. Universitat Politècnica de València, 46022. Valencia, Spain. E-mail: njthome@mat.upv.es.
} 
Bai in [2]. By generalizing centrosymmetric matrices, centrohermitian matrices are defined as those matrices $A \in \mathbb{C}^{m \times n}$ satisfying $J_{m} A J_{n}=\bar{A}$, where $\bar{A}$ stands for the conjugate of the corresponding entries of $A$. Each square centrohermitian matrix is similar to a matrix with real entries and a full information about the spectral properties of square centrohermitian matrices is given, for instance, in [12]. Moreover, $m$-involutions (i.e., $K^{m}=I$ ) that commute (and anti-commute) with a diagonalizable matrix were studied in [17].

In addition, a more general situation where the equation $K A=A^{s+1} K$ is studied for involutory matrices $K$ has been analyzed in $[8,10]$ for nonnegative integer values of $s$. They are called $\{K, s+1\}$-potent matrices and when $s=0$ it is called a $\{K\}$-centrosymmetric matrix. Complementing this study, the case $s=-2$, that corresponds to generalized centro-invertible matrices, has been considered in [11]. In addition, some applications for image blurring/deblurring were developed. Clearly, $\{K, s+1\}$-potent matrices generalize centrosymmetric ones to a wider class of matrices.

On the other hand, the sign function is studied in [7]. It is observed that in general, $\operatorname{sign}(A)$ is a nonprimary square root of the identity. However, the square root can be obtained from the sign function. It is also shown that the sign function has applications in several areas. For example, it plays an important role in iterative methods for matrix roots and the polar decomposition. Moreover, it is useful in applications to Riccati equations, the eigenvalue problem, and lattice quantum chromodynamics. Furthermore, it is well-known that every square matrix $A$ is $\{\operatorname{sign}(A)\}$-centrosymmetric.

This paper presents a deeper study of generalized centro-invertible matrices. In Section 2, we state the coordinability between the classes of involutory matrices, generalized centro-invertible matrices, and $\{K\}$-centrosymmetric matrices. Then, some characterizations of generalized centro-invertible matrices are obtained. In Section 3, a spectral study of generalized centro-invertible matrices is given. The inverse problem associated to generalized centro-invertible matrices is analyzed in Section 4. Section 5 is devoted to design some algorithms for solving both the direct and the inverse problem, and illustrating examples are presented. Section 6 studies the relation between generalized centro-invertible matrices and the sign function. In particular, we prove that the sign of a generalized centro-invertible matrix is $\{K\}$-centrosymmetric and that the class of generalized centro-invertible matrices is closed under the matrix sign function. Finally, Section 7 includes the form to extend our results to the problem $K A K=A^{-(s+1)}$.

\section{Generalized centro-invertible matrices}

In $[15,16]$, Wikramaratna studied all matrices $X$ such that $X^{-1}=X_{R}$, where $X_{R}=J X J$, for $J$ being the interchange matrix (that is, $J$ is the matrix with 1 's on the secondary diagonal and 0's otherwise). In that paper, the author focuses his study on matrices over the integers modulo $m$, since his goal was to apply them to cryptography. 
We recall that for a given involutory matrix $K \in \mathbb{C}^{n \times n}$, a matrix $A \in \mathbb{C}^{n \times n}$ is called generalized centro-invertible if $K A K=A^{-1}$ [11]. It is clear that this class of matrices is a subclass of nonsingular matrices with determinant \pm 1 .

Let $\mathbf{G C I} \mathbf{I}_{K}$ be the set defined by

$$
\mathbf{G C I}_{K}=\left\{A \in \mathbb{C}^{n \times n}: K A K=A^{-1}\right\} ;
$$

and let $\mathbf{I}, \mathbf{P} \subset \mathbb{C}^{n \times n}$ be the sets of all complex involutory and idempotent matrices, respectively.

Next, we relate these three sets.

Theorem 1 The sets $\mathbf{G C I}_{K}$, I, and $\mathbf{P}$ are equipotent.

Proof. It is easy to see that the map $\Psi: \mathbf{G C I}_{K} \rightarrow \mathbf{I}$ given by $A \mapsto K A$ is well-defined since $K A K=A^{-1}$ implies $[\Psi(A)]^{2}=(K A)^{2}=I_{n}$, so $\Psi(A) \in \mathbf{I}$. Moreover, it is immediate that $\Psi$ is one-to-one and surjective (because of the nonsingularity of $K$ and $\Psi\left(K^{-1} B\right)=B$ for every $\left.B \in \mathbf{I}\right)$. Thus, $\Psi$ is a bijection. Hence, $\mathbf{G C I}_{K} \simeq \mathbf{I}$.

Notice that, even more, it can be said: $\Psi$ is an involution since $\Psi^{2}(A)=$ $K(K A)=A$, for every $A \in \mathbf{G C I}_{K}$.

On the other hand, we consider the function $\xi: \mathbf{G C I}_{K} \rightarrow \mathbf{P}$ given by $\xi(A)=\frac{1}{2}\left(I_{n}-K A\right)$ for $A \in \mathbf{G C I}_{K}$. It is well-defined because $K A K=A^{-1}$ implies $\xi^{2}(A)=\frac{1}{4}\left(I_{n}-K A-K A+(K A)^{2}\right)=\frac{1}{2}\left(I_{n}-K A\right)=\xi(A)$. Moreover, injectiveness of $\xi$ is trivial and for $B \in \mathbf{G C I}_{K}$ and $A=K\left(I_{n}-2 B\right)$ we have $\xi(A)=B$. Hence, $\mathbf{G C I}_{K} \simeq \mathbf{P}$.

Remark 2 Notice that $\mathbf{G C I}_{K} \nsubseteq \mathbf{I}$ and $\mathbf{G C I} \mathbf{I}_{K} \nsubseteq \mathbf{P}$ as matrices

$$
A=\frac{1}{3}\left[\begin{array}{rr}
4 & 1 \\
-1 & 2
\end{array}\right] \quad \text { and } \quad K=\left[\begin{array}{ll}
0 & 1 \\
1 & 0
\end{array}\right]
$$

allow us to check. Similarly, $\mathbf{I} \nsubseteq \mathbf{G C I}_{K}$ as it can be verified with

$$
A=\left[\begin{array}{rr}
1 & 0 \\
0 & -1
\end{array}\right] \quad \text { and } \quad K=\left[\begin{array}{rr}
1 & 1 \\
0 & -1
\end{array}\right] .
$$

Finally, any idempotent singular matrix allows us to show that $\mathbf{P} \nsubseteq \mathbf{G C I}_{K}$. Hence, $\mathbf{G C I}_{K}, \mathbf{I}$, and $\mathbf{P}$ are equipotent classes of different each other sets.

Next we give a characterization of matrices in $\mathbf{G C I} \mathbf{I}_{K}$.

Theorem 3 Let $A \in \mathbb{C}^{n \times n}$. Then the following conditions are equivalent:

(a) $A \in \mathbf{G C I}_{K}$.

(b) $(K A)^{2}=I_{n}$.

(c) There exists a nonsingular matrix $P \in \mathbb{C}^{n \times n}$ such that

$$
A=K P\left[\begin{array}{cc}
I & O \\
O & -I
\end{array}\right] P^{-1} .
$$


(d) There exists an idempotent matrix $B \in \mathbb{C}^{n \times n}$ such that $K A+2 B=I_{n}$.

Proof. (a) $\Longleftrightarrow$ (b) Is easy to see by Definition.

(b) $\Longleftrightarrow(\mathrm{c})$ Is an immediate consequence from the fact $(K A)^{2}=I_{n}$ if and only if there exists a nonsingular matrix $P \in \mathbb{C}^{n \times n}$ such that

$$
K A=P\left[\begin{array}{cc}
I & O \\
O & -I
\end{array}\right] P^{-1} .
$$

(a) $\Longleftrightarrow$ (d) Follows from Theorem 1 .

The following result gives a necessary condition for $A$ to be in $\mathbf{G C I}_{K}$.

Proposition 4 If $A \in \mathbf{G C I}_{K}$ and $K A$ has $p$ eigenvalues equal to 1 then $\operatorname{trace}(K A) \leq n$. Moreover, $\operatorname{trace}(K A)>0$ (resp., $<0$ or $=0)$ if $p>n / 2$ (resp., $p<n / 2$ or $p=n / 2$ ).

Proof. By Theorem 3, there is $B \in \mathbb{C}^{n \times n}$ such that $B^{2}=B$ and $K A+2 B=I_{n}$. By taking the trace in both sides we have $\operatorname{trace}(K A)+2 \operatorname{trace}(B)=n$. Since $\operatorname{trace}(B)=\operatorname{rank}(B)$ we arrive at $\operatorname{rank}(B)=\frac{n-\operatorname{trace}(K A)}{2}$. Hence, $\operatorname{trace}(K A) \leq$ $n$. By counting 1's and -1 's in the representation (1) we obtain the sign of the trace of $K A$.

Despite $K A$ and $A K$ not being necessarily equal, a dual result to Theorem 3 can be stated for $A K$ instead of $K A$.

On the other hand, it is well known that for an involutory matrix $K \in \mathbb{C}^{n \times n}$, there exists a nonsingular matrix $P \in \mathbb{C}^{n \times n}$ such that

$$
K=P\left[\begin{array}{cc}
I & O \\
O & -I
\end{array}\right] P^{-1}
$$

Theorem 5 Let $A \in \mathbb{C}^{n \times n}$ and $K$ be factorized as in (2). Then $A \in \mathbf{G C I}_{K}$ if and only if there exist matrices $M, N, R$, and $S$ of appropriate sizes (for $M$ being a square matrix) such that

$$
A=P\left[\begin{array}{cc}
M & N \\
R & S
\end{array}\right] P^{-1}
$$

where the block matrices satisfy: (i) $M^{2}-N R=I$, (ii) $M N=N S$, (iii) $R M=S R$, and (iv) $S^{2}-R N=I$.

Proof. We start by writing $K$ as in (2) and considering the partition for $A$ given in (3). From Theorem 3, condition $(K A)^{2}=I_{n}$ yields

$$
\left[\begin{array}{cc}
M^{2}-N R & M N-N S \\
-R M+S R & -R N+S^{2}
\end{array}\right]=I_{n}
$$

Then conditions on $M, N, R$, and $S$ are necessary. The sufficiency of these conditions is evident taking into account that $(K A)^{2}=I_{n}$ implies the nonsingularity of $A$.

By $\sigma(A)$ we will denote the spectrum of $A \in \mathbb{C}^{n \times n}$. 
Corollary 6 Let $A \in \mathbb{C}^{n \times n}$ be such that $A \in \mathbf{G C I}_{K}$. The blocks $M, N, R$, and $S$ of Theorem 5 satisfy the following statements:

(a) $\operatorname{trace}\left(M^{2}\right)=\operatorname{trace}\left(N^{2}\right)$.

(b) $\operatorname{trace}\left(M^{3}\right)-\operatorname{trace}(M)=\operatorname{trace}\left(S^{3}\right)-\operatorname{trace}(S)=\operatorname{trace}(N S R)$.

(c) $\operatorname{det}(M)= \pm \operatorname{det}(S)$. Consequently, $M$ and $S$ are either both nonsingular or both singular.

(d) One of the following conditions holds:

(i) If $\alpha \in \sigma(M)$ then $\pm \alpha \in \sigma(S) \cup\{1\}$.

(ii) If $\beta \in \sigma(S)$ then $\pm \beta \in \sigma(M) \cup\{1\}$.

(e) $N S^{2}=M^{2} N$.

(f) $N R$ and $M$ commute as well as $R N$ and $S$.

Proof. (a) Subtracting (iv) from (i) in Theorem 5 and taking the trace we get $\operatorname{trace}\left(M^{2}\right)=\operatorname{trace}\left(N^{2}\right)$ since $\operatorname{trace}(N R)=\operatorname{trace}(R N)$.

(b) Postmultiplying $M^{2}-N R=I$ by $M, S^{2}-R N=I$ by $S$ and using that $\operatorname{trace}(N S R)=\operatorname{trace}(R N S)$ we get $(\mathrm{b})$.

(c) By (i), $(\operatorname{det}(M))^{2}=\operatorname{det}\left(M^{2}\right)=\operatorname{det}(I+N R)$. Similarly, by (iv), $(\operatorname{det}(S))^{2}=\operatorname{det}\left(S^{2}\right)=\operatorname{det}(I+R N)$. Since the Sylvester's formula guarantees that $\operatorname{det}(I+N R)=\operatorname{det}(I+R N)$, we $\operatorname{get} \operatorname{det}(M)= \pm \operatorname{det}(S)$.

(d) From (i) and (iv) we have $\sigma(N R)=\sigma\left(M^{2}-I\right)$ and $\sigma(R N)=\sigma\left(S^{2}-\right.$ $I)$, respectively. The matrices $R N \in \mathbb{C}^{s \times s}$ and $N R \in \mathbb{C}^{\ell \times \ell}$ have the "same spectrum" counting multiplicities (except for some additional eigenvalues equal to 0$)$. Without any loss of generality, we assume $s \geq \ell$. So, if $s=\ell$, for a given $\alpha \in \sigma(M)$, it is clear that $\alpha^{2}-1 \in \sigma\left(M^{2}-I\right)$. The Jordan canonical forms of $M$ and $S$ yield to $\alpha^{2}-1=\beta^{2}-1$ for some $\beta \in \sigma(S)$. In this case, $\beta= \pm \alpha$. If $s>\ell$ then again $\beta= \pm \alpha$ and, moreover, there exist $s-\ell$ eigenvalues $\alpha$ 's $\in\{-1,1\}$.

(e) Pre-multiplying (iv) by $N$, post-multiplying (i) by $N$, and then subtracting them we get $N S^{2}=M^{2} N$.

(f) Follows directly from (ii) and (iii).

Schur complement gives another approach to matrices in $\mathbf{G C I}_{K}$. For a nonsingular matrix $M$, we denote the Schur complement of $M$ in $Z$ by $Z / M=$ $S-R M^{-1} N$ where

$$
Z=\left[\begin{array}{cc}
M & N \\
R & S
\end{array}\right]
$$

for $S$ being also a square matrix. In this case, $\operatorname{det}(Z)=\operatorname{det}(M) \operatorname{det}(Z / M)$ and

$$
\left[\begin{array}{cc}
M & N \\
R & S
\end{array}\right]^{-1}=\left[\begin{array}{cc}
M^{-1}+M^{-1} N(Z / M)^{-1} R M^{-1} & -M^{-1} N(Z / M)^{-1} \\
-(Z / M)^{-1} R M^{-1} & (Z / M)^{-1}
\end{array}\right] .
$$

Analogously, the Schur complement of $S$ in $Z$ is $Z / S=M-N S^{-1} R$. 
Theorem 7 Let $K$ be factorized as in (2) and $A \in \mathbb{C}^{n \times n}$ such that

$$
A=P\left[\begin{array}{cc}
M & N \\
R & S
\end{array}\right] P^{-1}
$$

for some blocks $M, N, R$, and $S$ of adequate sizes. If $A \in \mathbf{G C I}_{K}$ then

(a) $M \neq O$ and $S \neq O$.

(b) only one of the following conditions holds:

(i) $M$ and $S$ are singular.

(ii) $M$ and $S$ are nonsingular, $M+N(Z / M)^{-1} R=M^{3}, R=(Z / M) R M$, $N=M N(Z / M),(Z / M) S=I, S+R(Z / S)^{-1} N=S^{3}, N=(Z / S) N S$, $R=S R(Z / S)$, and $(Z / S) M=I$.

Proof. (a) Assume that $M=O$. By Theorem 5 we get $N R=-I, N S=O$, $S R=O$, and $S^{2}-R N=I$. Multiplying the last equality by $S$ on the right side and using $N S=O$, we arrive at $S^{3}=S$. If $S$ is nonsingular then $R=O$, which contradicts $N R=-I$. So, $S$ is singular and has index 1. Thus, there exists a nonsingular matrix $P_{S}$ such that

$$
S=P_{S}\left[\begin{array}{ll}
D & O \\
O & O
\end{array}\right] P_{S}^{-1}
$$

where $D$ is a nonsingular (diagonal) matrix. Now we partition

$$
R=P_{S}\left[\begin{array}{ll}
R_{1} & R_{2} \\
R_{3} & R_{4}
\end{array}\right] P_{S}^{-1} \quad \text { and } \quad N=P_{S}\left[\begin{array}{cc}
N_{1} & N_{2} \\
N_{3} & N_{4}
\end{array}\right] P_{S}^{-1}
$$

according to the sizes of $S$. From $S R=O$ we get $R_{1}=O$ and $R_{2}=O$ and, from $N S=O$ we get $N_{1}=O$ and $N_{3}=O$. Since $N R=-I$, we have $N_{2} R_{3}=-I$ and $N_{4} R_{4}=-I$, that is, $R_{4}=-N_{4}^{-1}$. By substituting in $S^{2}-R N=I$ we arrive at $R_{3} N_{2}=O$. Multiplying this last equality on the right hand side by $R_{3}$, we obtain $R_{3}=O$, which contradicts $N_{2} R_{3}=-I$. Hence, $M \neq O$. Analogously, it can be shown that $S \neq O$.

(b) Since $A$ is nonsingular, if $M$ is nonsingular then $Z / M$ also is. Now, if we consider $K$ factorized as in (2),

$$
K A K=P\left[\begin{array}{rr}
M & -N \\
-R & S
\end{array}\right] P^{-1}
$$

and by means of the Banachiewicz-Schur formula (4), the equality $K A K=A^{-1}$ provides the conditions related to matrix $M$. Similarly, the conditions related to matrix $S$ can be stated.

Remark 8 It is easy to see that the conditions obtained in Theorem 5 are equivalent to those given in (b) of Theorem $\%$. 


\section{Spectral analysis of matrices in $\mathbf{G C I}_{K}$}

This section studies the spectral analysis of generalized centro-invertible matrices. On the one hand, we recall that for a given matrix in $A \in \mathbf{G C I}_{K}$, the following fact is true: $\sigma(K A)=\sigma(A K) \subseteq\{-1,1\}$. On the other hand, by using properties of the trace function, if $K A K=A^{-1}$ then $\operatorname{trace}\left(A^{-1}\right)=$ $\operatorname{trace}(K(A K))=\operatorname{trace}((A K) K)=\operatorname{trace}(A)$ and then

$$
\sum_{i=1}^{n} \lambda_{i}=\sum_{i=1}^{n} \lambda_{i}^{-1}
$$

where $\sigma(A)=\left\{\lambda_{1}, \ldots, \lambda_{n}\right\}$. This information can be more detailed as we obtain in the following result.

Theorem 9 Let $A \in \mathbb{C}^{n \times n}$ be such that $A \in \mathbf{G C I}_{K}$. Then there exist nonzero complex scalars $\lambda_{1}, \ldots, \lambda_{p}$ such that

$$
\sigma(A) \subseteq\left\{\lambda_{1}, \lambda_{1}^{-1}, \ldots, \lambda_{p}, \lambda_{p}^{-1}, 1,-1\right\}
$$

with

(a) $\lambda_{i} \neq \lambda_{j}$ for $i \neq j, i, j=1, \ldots, p$.

(b) $\lambda_{i} \neq \lambda_{i}^{-1}$ for $i=1, \ldots, p$.

(c) For $i=1, \ldots, p, \lambda_{i} \in \sigma(A) \Longleftrightarrow \lambda_{i}^{-1} \in \sigma(A)$.

Proof. Since similar matrices have the same spectrum, if $A \in \mathbf{G C I}_{K}$ then $\sigma(A)=\sigma\left(A^{-1}\right)$. Now, if $\lambda \in \sigma(A)$ then two situations may occur:

- $\lambda^{-1}=\lambda$. Hence, $\lambda= \pm 1$.

- $\lambda^{-1} \neq \lambda$. Then, there exists $\mu \in \sigma(A)-\{\lambda\}$ such that $\lambda^{-1}=\mu$. That is, if $\lambda \in \sigma(A)=\sigma\left(A^{-1}\right)$ then it is clear that $\lambda^{-1} \in \sigma(A)$. Hence, both $\lambda, \lambda^{-1} \in \sigma(A)$.

Remark 10 Notice that Theorem 9 it is not saying that the eigenvalues $\lambda$ 's of A must have algebraic multiplicity one. By considering the matrices

$$
A=\left[\begin{array}{cccc}
a & 0 & 0 & 0 \\
0 & 1 / a & 0 & 0 \\
0 & 0 & a & 0 \\
0 & 0 & 0 & 1 / a
\end{array}\right] \quad \text { and } \quad K=\left[\begin{array}{cccc}
0 & 1 & 0 & 0 \\
1 & 0 & 0 & 0 \\
0 & 0 & 0 & 1 \\
0 & 0 & 1 & 0
\end{array}\right]
$$

where $a \in \mathbb{C}-\{0, \pm 1\}$, we have an example of a matrix $A \in \mathbf{G C I}_{K}$ with a and $1 /$ a being repeated eigenvalues.

Moreover, nothing may be said about the algebraic multiplicity of 1 and -1 when they belong to $\sigma(A)$. Particularizing to $a=1$ or $a=-1$ the matrix $A$ above, we obtain a simple example for this last situation. 
In [8], it was shown that for a $\{K, s+1\}$-potent matrix $A$ (that is, $K A K=$ $A^{s+1}$, with $s \geq 1$ ), it follows that $A^{(s+1)^{2}}=A$. This fact was crucial to show that, in that case, $A$ is diagonalizable. Now, our question is the following: Is it possible to find a power $t \in \mathbb{N}$ such that $A^{t+1}=A$ if $A$ belongs to $\mathbf{G C I}_{K}$ ? The answer is negative as we can see in the following example. The matrix

$$
A=\frac{1}{3}\left[\begin{array}{rr}
4 & 1 \\
-1 & 2
\end{array}\right]
$$

belongs to $\mathbf{G C I}_{K}$ for

$$
K=\left[\begin{array}{ll}
0 & 1 \\
1 & 0
\end{array}\right]
$$

However, it is possible to show by induction that

$$
A^{t+1}-A=\frac{t}{3}\left[\begin{array}{rr}
1 & 1 \\
-1 & -1
\end{array}\right] \neq O, \text { for all } t \in \mathbb{N} .
$$

Even more, $A$ is not diagonalizable since its Jordan canonical form is given by

$$
A=\left[\begin{array}{rr}
\frac{1}{3} & 1 \\
-\frac{1}{3} & 0
\end{array}\right]\left[\begin{array}{ll}
1 & 1 \\
0 & 1
\end{array}\right]\left[\begin{array}{rr}
0 & -3 \\
1 & 1
\end{array}\right] \text {. }
$$

With the intention of finding a similar result to [8, Theorem 2], it is necessary to investigate how to relate matrices in $\mathbf{G C I}_{K}$ to diagonalization. Although a matrix may be in $\mathbf{G C I}_{K}$ not being diagonalizable, a similar relation as the aforementioned in [8] by using the projectors of a spectral decomposition of $A$ can be established in case of $A$ being diagonalizable.

Before doing that, we recall the following well-known result. Here, $\delta_{i j}$ represents the Kronecker delta.

Lemma 11 [3] Let $A \in \mathbb{C}^{n \times n}$ with $k$ distinct eigenvalues $\lambda_{1}, \ldots, \lambda_{k}$. Then $A$ is diagonalizable if and only if there exist disjoint projectors $P_{1}, P_{2}, \ldots, P_{k}$, that is $P_{i} P_{j}=\delta_{i j} P_{i}$ for $i, j \in\{1,2, \ldots, k\}$, such that $A=\sum_{j=1}^{k} \lambda_{j} P_{j}$ and $I_{n}=\sum_{j=1}^{k} P_{j}$. Moreover, these projectors are uniquely determined.

Theorem 12 Let $A \in \mathbb{C}^{n \times n}$ be a matrix in $\mathbf{G C I}_{K}$. If $A$ is diagonalizable with spectrum as in Theorem 9 then

(i) $K P_{i} K=\widetilde{P}_{i}$ for all $i=1, \ldots, p$.

(ii) $K P_{j} K=P_{j}$ for all $j \in\{p+1, p+2\}$.

where $P_{1}, \widetilde{P}_{1}, \ldots, P_{p}, \widetilde{P}_{p}, P_{p+1}, P_{p+2}$ are the projectors associated to the eigenvalues $\lambda_{1}, \lambda_{1}^{-1}, \ldots, \lambda_{p}, \lambda_{p}^{-1}, 1,-1$, respectively (see Lemma 11).

Proof. By hypothesis, matrix $A$ can be expressed as

$$
\begin{aligned}
A & =P \operatorname{diag}\left(\lambda_{1} I_{r_{1}}, \lambda_{1}^{-1} I_{r_{1}}, \ldots, \lambda_{p} I_{r_{p}}, \lambda_{p}^{-1} I_{r_{p}}, I_{r_{p+1}},-I_{r_{p+2}}\right) P^{-1} \\
& =\lambda_{1} P_{1}+\lambda_{1}^{-1} \widetilde{P}_{1}+\cdots+\lambda_{p} P_{p}+\lambda_{p}^{-1} \widetilde{P}_{p}+P_{p+1}-P_{p+2},
\end{aligned}
$$


where $P_{1}, \widetilde{P}_{1}, \ldots, P_{p}, \widetilde{P}_{p}, P_{p+1}, P_{p+2}$ are the projectors associated to the eigenvalues $\lambda_{1}, \lambda_{1}^{-1}, \ldots, \lambda_{p}, \lambda_{p}^{-1}, 1,-1$, respectively. Without loss of generality, we assume that all of these eigenvalues are in the spectrum. It is then clear that the inverse of matrix $A$ is given by

$$
A^{-1}=\lambda_{1}^{-1} P_{1}+\lambda_{1} \widetilde{P}_{1}+\cdots+\lambda_{p}^{-1} P_{p}+\lambda_{p} \widetilde{P}_{p}+P_{p+1}-P_{p+2} .
$$

Since $A \in \mathbf{G C I}_{K}$, from $K A K=A^{-1}$ we get

$$
\begin{aligned}
K P_{1} K & =\widetilde{P}_{1}, \\
& \vdots \\
K P_{p} K & =\widetilde{P}_{p}, \\
K P_{p+1} K & =P_{p+1}, \\
K P_{p+2} K & =P_{p+2} .
\end{aligned}
$$

For $i=1, \ldots, p, p+1, p+2$ we have $\left(K P_{i} K\right)^{2}=K P_{i}^{2} K=K P_{i} K$, from where every $K P_{i} K$ is idempotent.

Example 13 By applying Theorem 12 to the diagonal matrix

$$
A=\left[\begin{array}{cc}
2 & 0 \\
0 & 1 / 2
\end{array}\right]=2\left[\begin{array}{ll}
1 & 0 \\
0 & 0
\end{array}\right]+\frac{1}{2}\left[\begin{array}{ll}
0 & 0 \\
0 & 1
\end{array}\right],
$$

where the projector associated to the eigenvalue 2 is called $P_{1}$ and the corresponding to $1 / 2$ is $\widetilde{P}_{1}$. Clearly, $\sigma(A)=\{2,1 / 2\}$ and $K P_{1} K=\widetilde{P}_{1}$ for

$$
K=\left[\begin{array}{ll}
0 & 1 \\
1 & 0
\end{array}\right] .
$$

Hence, $A \in \mathbf{G C I}_{K}$.

We close this section with the following two properties: If $A \in \mathbb{C}^{n \times n}$ is nonsingular and $K \in \mathbb{C}^{n \times n}$ is involutory then:

(a) $P A P^{-1} \in \mathbf{G C I}_{K}$ if and only if $A \in \mathbf{G C I}_{P^{-1} K P}$ for all nonsingular $P \in$ $\mathbb{C}^{n \times n}$.

(b) $A \in \mathbf{G C I}_{K}$ if and only if $P A P^{-1} \in \mathbf{G C I}_{P K P^{-1}}$ for all nonsingular $P \in$ $\mathbb{C}^{n \times n}$.

Clearly, those properties extend examples like the last one from diagonal to diagonalizable matrices. 


\section{The inverse problem}

Up to now, we have treated the problem of studying matrices $A$ such that $K A K=A^{-1}$ for a given involutory matrix $K$. This section is devoted to the inverse problem, that is, to find matrices $K$ satisfying the previous matrix relation for a given matrix $A$.

The next result gives explicit representations for the involutory matrices $K$ such that a given matrix $A$ be in $\mathbf{G C I} \mathbf{I}_{K}$.

Theorem 14 Let $A \in \mathbb{C}^{n \times n}$ be a diagonalizable matrix (i.e., $A=P D P^{-1}$, with $P$ nonsingular). Then there exists an involutory matrix $K$ such that $A$ belongs to $\mathbf{G C I}_{K}$ with spectrum as in Theorem 9 if and only if

$$
K=P\left(\left[\begin{array}{cc}
O & K_{1} \\
K_{1}^{-1} & O
\end{array}\right] \oplus \cdots \oplus\left[\begin{array}{cc}
O & K_{p} \\
K_{p}^{-1} & O
\end{array}\right] \oplus K_{p+1} \oplus K_{p+2}\right) P^{-1},
$$

for some arbitrary nonsingular matrices $K_{1}, \ldots, K_{p}$ of sizes $r_{1} \times r_{1}, \ldots, r_{p} \times r_{p}$, respectively, $K_{p+1}^{2}=I_{r_{p+1}}$ and $K_{p+2}^{2}=I_{r_{p+2}}$.

Proof. Assume that $A \in \mathbb{C}^{n \times n}$ is a diagonalizable matrix and that there exists an involutory matrix $K \in \mathbb{C}^{n \times n}$ such that $A \in \mathbf{G C I}_{K}$. The proof will be carried out by considering firstly some special cases. By applying Theorem 9 we know that every eigenvalue $\lambda_{i}$ has the same algebraic multiplicity as its inverse $\lambda_{i}^{-1}$.

- Case 1: $A$ has two nonzero distinct eigenvalues $\lambda$ and $\mu$ and their inverses. In this case,

$$
A=P\left[\begin{array}{cccc}
\lambda I_{r_{1}} & O & O & O \\
O & \lambda^{-1} I_{r_{1}} & O & O \\
O & O & \mu I_{r_{2}} & O \\
O & O & O & \mu^{-1} I_{r_{2}}
\end{array}\right] P^{-1}, \quad \lambda \neq \mu .
$$

The matrix $A$ can be written as

$$
A=\lambda P_{1}+\lambda^{-1} P_{2}+\mu P_{3}+\mu^{-1} P_{4},
$$

where $P_{i}=P E_{i} P^{-1}, E_{1}=\operatorname{diag}\left(I_{r_{1}}, O, O, O\right), E_{2}=\operatorname{diag}\left(O, I_{r_{1}}, O, O\right)$, $E_{3}=\operatorname{diag}\left(O, O, I_{r_{2}}, O\right)$, and $E_{4}=\operatorname{diag}\left(O, O, O, I_{r_{2}}\right)$.

The inverse of $A$ is given by

$$
A^{-1}=\lambda^{-1} P_{1}+\lambda P_{2}+\mu^{-1} P_{3}+\mu P_{4} .
$$

From $K A K=A^{-1}$ we have that $\lambda K P_{1} K+\lambda^{-1} K P_{2} K+\mu K P_{3} K+$ $\mu^{-1} K P_{4} K=\lambda^{-1} P_{1}+\lambda P_{2}+\mu^{-1} P_{3}+\mu P_{4}$. The uniqueness of the spectral decomposition gives $K P_{1} K=P_{2}$ and $K P_{3} K=P_{4}$. It is trivial that $K P_{i} K$ is idempotent. 
Now we denote $M=P^{-1} K P$. By multiplying $K P_{1}=P_{2} K$ by $P^{-1}$ on the left hand side and by $P$ on the right hand side we obtain

$$
M\left[\begin{array}{cccc}
I_{r_{1}} & O & O & O \\
O & O & O & O \\
O & O & O & O \\
O & O & O & O
\end{array}\right]=\left[\begin{array}{cccc}
O & O & O & O \\
O & I_{r_{1}} & O & O \\
O & O & O & O \\
O & O & O & O
\end{array}\right] M
$$

where $M$ is partitioned in blocks of adequate sizes as follows

$$
M=\left[\begin{array}{llll}
M_{11} & M_{12} & M_{13} & M_{14} \\
M_{21} & M_{22} & M_{23} & M_{24} \\
M_{31} & M_{32} & M_{33} & M_{34} \\
M_{41} & M_{42} & M_{43} & M_{44}
\end{array}\right]
$$

We can easily obtain $M_{11}=O, M_{31}=O, M_{41}=O, M_{22}=O, M_{23}=O$, and $M_{24}=O$. By substituting them in $M$ and proceeding in a similar way with $K P_{3}=P_{4} K$ we arrive at $M_{13}=O, M_{33}=O, M_{42}=O$, and $M_{44}=O$. Then

$$
K=P\left[\begin{array}{cccc}
O & M_{12} & O & M_{14} \\
M_{21} & O & O & O \\
O & M_{32} & O & M_{34} \\
O & O & M_{43} & O
\end{array}\right] P^{-1}
$$

Since $K$ is involutory,

$$
K=P\left[\begin{array}{cccc}
O & M_{12} & O & O \\
M_{12}^{-1} & O & O & O \\
O & O & O & M_{34} \\
O & O & M_{34}^{-1} & O
\end{array}\right] P^{-1} .
$$

- Case 2: $A$ has a nonzero eigenvalue as well as its inverse and, moreover, the eigenvalues 1 and -1 . As in Case $1, \lambda$ and $\lambda^{-1}$ have the same algebraic multiplicity. Thus,

$$
A=P\left[\begin{array}{cccc}
\lambda I_{r_{1}} & O & O & O \\
O & \lambda^{-1} I_{r_{1}} & O & O \\
O & O & I_{r_{2}} & O \\
O & O & O & -I_{r_{3}}
\end{array}\right] P^{-1} .
$$

Again, we write $M=P^{-1} K P$ and partition

$$
M=\left[\begin{array}{llll}
M_{11} & M_{12} & M_{13} & M_{14} \\
M_{21} & M_{22} & M_{23} & M_{24} \\
M_{31} & M_{32} & M_{33} & M_{34} \\
M_{41} & M_{42} & M_{43} & M_{44}
\end{array}\right] .
$$


Now, proceeding similarly as in Case 1 we get

$$
K=P\left[\begin{array}{cccc}
O & M_{12} & O & O \\
M_{12}^{-1} & O & O & O \\
O & O & M_{34} & O \\
O & O & O & M_{44}
\end{array}\right] P^{-1} .
$$

Now, it is clear that the general case can be obtained by a combination of the cases studied previously.

The converse is trivial.

Some examples illustrating the solution of the inverse problem will be given in the next section.

\section{Algorithms and numerical examples}

The algorithms designed in this section can easily be implemented on a computer. We have used the MATLAB package R2016a version 9.0. We will present some numerical examples in order to show the performance of our algorithms and demonstrate their applicability. The computational cost of all our Algorithms is $O\left(n^{3}\right)$.

The first algorithm solves the direct problem from a numerical point of view. In other words, this is a procedure to obtain examples of generalized centro-invertible matrices in a very easy way.

Algorithm 1

Input: An involutory matrix $K \in \mathbb{C}^{n \times n}$.

Output: A matrix $A$ belonging to $\mathbf{G C I}_{K}$.

Step 1 Generate an arbitrary nonsingular matrix $P \in \mathbb{C}^{n \times n}$.

Step 2 Fix the number of 1's corresponding to the parameter $r$; the number of -1 's is $n-r$.

Step 3 Compute the matrix $A=K P\left[\begin{array}{cc}I_{r} & O \\ O & -I_{n-r}\end{array}\right] P^{-1}$.

End.

Example 15 For the given involutory matrix

$$
K=\frac{1}{2}\left[\begin{array}{lll}
1 & 0 & 0 \\
0 & 0 & 1 \\
0 & 1 & 0
\end{array}\right],
$$

Algorithm 1 has randomly generated the nonsingular matrix

$$
P=\frac{1}{2}\left[\begin{array}{rrr}
-2 & -1 & 1 \\
2 & 0 & 1 \\
-4 & -1 & -4
\end{array}\right]
$$


and then the following generalized centro-invertible matrix is obtained

$$
A=\frac{1}{2}\left[\begin{array}{rrr}
1 & 1 & 1 \\
4 & -4 & -2 \\
-1 & 3 & 1
\end{array}\right] .
$$

Next, for a given nonsingular matrix $A \in \mathbb{C}^{n \times n}$ we wonder about the existence of an involutory matrix $K \in \mathbb{C}^{n \times n}$ such that $A \in \mathbf{G C I}_{K}$. Theorem 14 gives the response for diagonalizable matrices.

Consider the following example. For the given matrix

$$
A=\left[\begin{array}{ll}
1 & 1 \\
1 & 0
\end{array}\right]
$$

it would exist a matrix $K$ such that $K A K=A^{-1}$ if and only if $I_{n} K A=A^{-1} K I_{n}$ which is equivalent to

$$
\left(A^{T} \otimes I_{n}-I_{n} \otimes A^{-1}\right) v(K)=0,
$$

where $A^{T}$ denotes the transpose of $A$ and $v(K)$ the column vector obtained by stacking the columns of the matrix $K$ on top of one another (see [3]). In this example, by making some computations it is easily seen that its unique solution is $K=O$, which is not involutory. So, not always such a matrix $K$ exists.

However, this procedure allows us to construct an algorithm avoiding the use of the diagonalization which, furthermore, allows us to drop out the hypothesis of diagonalization extending Theorem 14 to the general case.

\section{Algorithm 2}

Input: A matrix $A \in \mathbb{C}^{n \times n}$ for some integer $n \geq 2$.

Outputs: All the involutory matrices $K \in \mathbb{C}^{n \times n}$ such that $A \in$ $\mathbf{G C I}_{K}$, if any such $K$ exist.

Step 1 Compute $A^{-1}$ by solving linear systems $A x=e_{i}$, where $e_{i}$ are the canonical vectors of $\mathbb{C}^{n}$.

Step 2 Construct the matrix $M:=A^{T} \otimes I_{n}-I_{n} \otimes A^{-1}$.

Step 3 Find the general solution $v$ to $M v=\mathbf{0}$. The $n^{2} \times 1$ vector $v$ will depend on $d=\operatorname{dim}(\operatorname{ker}(M))$ arbitrary parameters.

Step 4 If $v=\mathbf{0}$, or equivalently, if $d=0$, then go to Step 8 .

Step 5 Treating $v$ as $v=v(K)$ for an $n \times n$ complex matrix $K$ containing $d$ parameters, recover $K$ from $v$.

Step 6 Determine the allowed values for the $d$ arbitrary parameters so that $K^{2}=I_{n}$. If there are no allowed parameter values, then go to Step 8. 
Step 7 The output is the set of all of the matrices $K$ whose parameter values are allowed. Go to End.

Step 8 "There is no involutory matrix $K \in \mathbb{C}^{n \times n}$ such that $A \in$ $\mathbf{G C I}_{K} . "$

\section{End}

The following is an example of a matrix $A$ with repeated eigenvalues of a diagonalizable matrix.

Example 16 Let

$$
A=\left[\begin{array}{cccc}
2+2 i & \frac{7}{4}+\frac{9}{4} i & 0 & \frac{7}{4}+\frac{9}{4} i \\
0 & 2+2 i & 0 & 0 \\
0 & \frac{7}{4}+\frac{9}{4} i & \frac{1}{4}-\frac{1}{4} i & 0 \\
0 & -\frac{7}{4}-\frac{9}{4} i & 0 & \frac{1}{4}-\frac{1}{4} i
\end{array}\right]
$$

with $\left.\sigma(A)=\left\{2+2 i, \frac{1}{4}-\frac{1}{4} i\right)\right\}$ both with algebraic multiplicity equals to 2. All the matrices $K$ such that $A \in \mathbf{G C I}_{K}$ are given by Algorithm 2 as follows:

$$
K=\left[\begin{array}{cccc}
1 & 0 & -2 z-2 w-z w-4 & -(z+1)(w+2) \\
0 & 1 & -z-2 & -z-1 \\
\frac{z+1}{z+2} & -w \frac{z+1}{z+2} & -z-2 & -\frac{(z+1)^{2}}{z+2} \\
-1 & w & z+2 & z
\end{array}\right]
$$

where $z, w \in \mathbb{C}$ with $z \neq-2$.

As a variant of the algorithm given above we present the following one for diagonalizable matrices.

Algorithm 3

Input: A diagonalizable matrix $A \in \mathbb{C}^{n \times n}$ for some $n \geq 2$.

Outputs: All the involutory matrices $K \in \mathbb{C}^{n \times n}$ such that $A \in$ $\mathbf{G C I}_{K}$, if any such $K$ exist.

Step 1 Diagonalize $A=P D P^{-1}$.

Step 2 Construct $M:=I_{n} \otimes D^{s+1}-D \otimes I$.

Step 3 Find the general solution $v$ to $M v=\mathbf{0}$. The $n^{2} \times 1$ vector $v$ will depend on $d=\operatorname{dim}(\operatorname{ker}(M))$ arbitrary parameters.

Step 4 If $v=\mathbf{0}$, or equivalently, if $d=0$, then go to Step 8 .

Step 5 Treating $v$ as $v=v(K)$ for an $n \times n$ complex matrix $K$ containing $d$ parameters, recover $K$ from $v$.

Step 6 Determine the allowed values for the $d$ arbitrary parameters so that $K^{2}=I_{n}$. If there are no allowed parameter values, then go to Step 8. 
Step 7 The output is the set of all of the matrices $K$ whose parameter values are allowed. Go to End.

Step 8 "There is no involutory matrix $K \in \mathbb{C}^{n \times n}$ such that $A \in$ $\mathbf{G C I}_{K}$."

\section{End}

We consider an example of a non-diagonalizable matrix with repeated eigenvalues.

Example 17 Consider the matrix

$$
A=\left[\begin{array}{cccc}
2 & 1 & 0 & 0 \\
0 & 2 & 0 & 0 \\
0 & 0 & 1 / 2 & 1 \\
0 & 0 & 0 & 1 / 2
\end{array}\right] .
$$

Algorithm 3 gives the following matrices $K$ such that $K A K=A^{-1}$ :

$$
K=\left[\begin{array}{cccc}
0 & 0 & -\frac{1}{4 z} & \frac{w}{4 z^{2}} \\
0 & 0 & 0 & \frac{1}{z} \\
-4 z & w & 0 & 0 \\
0 & z & 0 & 0
\end{array}\right]
$$

for both arbitrary $z \in \mathbb{C}-\{0\}$ and $w \in \mathbb{C}$.

Finally, we consider an example of a non-diagonalizable matrix $A$ having the distinguished eigenvalues 1's.

Example 18 By applying Algorithm 3 to the matrix

$$
A=\frac{1}{3}\left[\begin{array}{rr}
4 & 1 \\
-1 & 2
\end{array}\right]
$$

we get the following involutory matrices such that $A \in \mathbf{G C I}_{K}$ :

$$
K=\left[\begin{array}{cc}
-z & -z-1 \\
z-1 & z
\end{array}\right]
$$

with arbitrary $z \in \mathbb{C}$.

\section{Relation with the matrix sign function}

Throughout this section, $S \in \mathbb{C}^{n \times n}$ is assumed to have no eigenvalues on the imaginary axis. Hence, $S$ is nonsingular and the matrix sign function will be well-defined.

As it was defined by Higham in [7], if $S=Z J Z^{-1}$ is a Jordan canonical form arranged so that $J=\operatorname{diag}\left(J_{1}, J_{2}\right)$, where the eigenvalues of $J_{1} \in \mathbb{C}^{p \times p}$ lie 
in the open left half-plane and those of $J_{2} \in \mathbb{C}^{(n-p) \times(n-p)}$ lie in the open right half-plane, then

$$
\operatorname{sign}(S):=Z\left[\begin{array}{rc}
-I_{p} & O \\
O & I_{n-p}
\end{array}\right] Z^{-1} .
$$

Notice that in general $A K \neq K A$ for a matrix $A \in \mathbf{G C I}_{K}$. A first property is given in the following result.

Theorem 19 If $A \in \mathbf{G C I}_{K}$ then $K A$ and $A K$ are fixed points of the matrix sign function.

Proof. By Theorem 3, if $A \in \mathbf{G C I}_{K}$ then $K A$ is similar to the direct sum $\operatorname{diag}\left(-I_{p}, I_{n-p}\right)$, that is

$$
K A=P\left[\begin{array}{rc}
-I_{p} & O \\
O & I_{n-p}
\end{array}\right] P^{-1}
$$

for some nonsingular matrix $P \in \mathbb{C}^{n \times n}$. So, $K A$ has no eigenvalues on the imaginary axis. Now, it is evident that $\operatorname{sign}(K A)=K A$ holds. The proof is similar for $A K$.

We now prove that the sign of a generalized centro-invertible matrix is a $\{K\}$-centrosymmetric matrix and that the class of generalized centro-invertible matrices is closed under the matrix sign function.

Theorem 20 Let $A \in \mathbb{C}^{n \times n}$ be such that $A \in \mathbf{G C I}_{K}$ and $A$ has no eigenvalues on the imaginary axis. Then:

(a) the sign of $A$ is a $\{K\}$-centrosymmetric matrix.

(b) $\operatorname{sign}(A) \in \mathbf{G C I}_{K}$.

Proof. By the assumption, for some nonsingular matrix $P \in \mathbb{C}^{n \times n}$ we have

$$
A=P\left[\begin{array}{cc}
J_{1} & O \\
O & J_{2}
\end{array}\right] P^{-1}
$$

where $J_{1} \in \mathbb{C}^{p \times p}, J_{2} \in \mathbb{C}^{(n-p) \times(n-p)}$, and

$$
\sigma\left(J_{1}\right) \subseteq\{z \in \mathbb{C}: \operatorname{Real}(z)<0\}, \quad \sigma\left(J_{2}\right) \subseteq\{z \in \mathbb{C}: \operatorname{Real}(z)>0\} .
$$

Clearly,

$$
A^{-1}=P\left[\begin{array}{cc}
J_{1}^{-1} & O \\
O & J_{2}^{-1}
\end{array}\right] P^{-1} .
$$

For a nonzero complex number $\alpha+i \beta$ we have

$$
\operatorname{Real}\left(\frac{1}{\alpha+i \beta}\right)=\frac{\alpha}{\alpha^{2}+\beta^{2}}
$$


and so we get

$$
\sigma\left(J_{1}^{-1}\right) \subseteq\{z \in \mathbb{C}: \operatorname{Real}(z)<0\}, \quad \sigma\left(J_{2}^{-1}\right) \subseteq\{z \in \mathbb{C}: \operatorname{Real}(z)>0\} .
$$

Then,

$$
\operatorname{sign}(A)=P\left[\begin{array}{rc}
-I_{p} & O \\
O & I_{n-p}
\end{array}\right] P^{-1}=\operatorname{sign}\left(A^{-1}\right)
$$

Moreover, from $A^{-1}=K A K$ we obtain $\operatorname{sign}\left(A^{-1}\right)=K \operatorname{sign}(A) K$. Hence, $\operatorname{sign}(A) K=K \operatorname{sign}(A)$ because $K^{2}=I_{n}$. So, (a) holds.

Item (b) follows from (a) and using the property $\operatorname{sign}\left(A^{-1}\right)=[\operatorname{sign}(A)]^{-1}$.

Remark 21 Notice that the converse of Theorem 20 is not valid because sign(.) is not a one-to-one function as it can be seen in the following example.

Let

$$
A=\left[\begin{array}{cccc}
-1+i & -\frac{1}{2}+\frac{3}{2} i & -2+i & -\frac{1}{4}+\frac{3}{4} i \\
0 & 1 & 0 & 0 \\
0 & 0 & 1 & 0 \\
0 & -3-i & 0 & -\frac{1}{2}-\frac{1}{2} i
\end{array}\right]
$$

and the involutory matrix

$$
K=\left[\begin{array}{rrrr}
1 & 3 & 3 & 0 \\
0 & 2 & 1 & 0 \\
0 & -3 & -2 & 0 \\
2 & -6 & 0 & -1
\end{array}\right]
$$

$A$ Jordan canonical form of $A$ is given by $A=P J P^{-1}$ where

$$
J=\left[\begin{array}{ccrcc}
-1+i & 0 & 0 & 0 \\
0 & -\frac{1}{2}-\frac{1}{2} i & 0 & 0 \\
0 & 0 & 1 & 0 \\
0 & 0 & 0 & 1
\end{array}\right], \quad P=\left[\begin{array}{rrrr}
1 & 1 & -1 & 0 \\
0 & 0 & 0 & 1 \\
0 & 0 & 1 & 0 \\
0 & -2 & 0 & -2
\end{array}\right]
$$

Then

$$
\operatorname{sign}(A)=P\left[\begin{array}{rrrr}
-1 & 0 & 0 & 0 \\
0 & -1 & 0 & 0 \\
0 & 0 & 1 & 0 \\
0 & 0 & 0 & 1
\end{array}\right] P^{-1}=\left[\begin{array}{rrrr}
-1 & 0 & -2 & 0 \\
0 & 1 & 0 & 0 \\
0 & 0 & 1 & 0 \\
0 & -4 & 0 & -1
\end{array}\right] .
$$

We have $K \operatorname{sign}(A)=\operatorname{sign}(A) K$ but

$$
(K A)^{2}=\left[\begin{array}{cccc}
-1-4 i & -4-3 i & -2-4 i & -2-\frac{3}{2} i \\
0 & 1 & 0 & 0 \\
0 & 0 & 1 & 0 \\
-4-8 i & -12-4 i & -4-8 i & -5-2 i
\end{array}\right] \neq I_{4}
$$

which proves that $A \notin \mathbf{G C I}_{K}$. 
The following example illustrates the results obtained in this section.

Example 22 Let $A$ be the same matrix as in Remark 21 and the involutory matrix

$$
K=\left[\begin{array}{rrrr}
\frac{1}{2} & -\frac{3}{2} & -\frac{1}{2} & -\frac{3}{4} \\
0 & 1 & 0 & 0 \\
0 & 0 & 1 & 0 \\
-1 & -3 & -1 & -\frac{1}{2}
\end{array}\right]
$$

Then, $A \in \mathbf{G C I}_{K}$ and by Theorem 19

$$
\operatorname{sign}(K A)=K A=\left[\begin{array}{cccc}
-\frac{1}{2}+\frac{1}{2} i & \frac{1}{2}+\frac{3}{2} i & -\frac{3}{2}+\frac{1}{2} i & \frac{1}{4}+\frac{3}{4} i \\
0 & 1 & 0 & 0 \\
0 & 0 & 1 & 0 \\
1-i & -1-i & 1-i & \frac{1}{2}-\frac{1}{2} i
\end{array}\right]
$$

It can be also checked that $K \operatorname{sign}(A)=\operatorname{sign}(A) K$ and $\operatorname{sign}(A) \in \mathbf{G C I}_{K}$ as Theorem 20 assures and, moreover, a Jordan canonical form for $K A$ is given by

$K A=\left[\begin{array}{cccc}\frac{3}{4}-\frac{1}{4} i & \frac{1}{4}+\frac{1}{4} i & -1 & i \\ 0 & 0 & 0 & 1 \\ 0 & 0 & 1 & 0 \\ -\frac{1}{2}+\frac{1}{2} i & \frac{1}{2}-\frac{1}{2} i & 0 & 0\end{array}\right]\left[\begin{array}{rrrr}-1 & 0 & 0 & 0 \\ 0 & 1 & 0 & 0 \\ 0 & 0 & 1 & 0 \\ 0 & 0 & 0 & 1\end{array}\right]\left[\begin{array}{cccc}1 & -i & 1 & -\frac{1}{2} i \\ 1 & -i & 1 & 1+\frac{1}{2} i \\ 0 & 0 & 1 & 0 \\ 0 & 1 & 0 & 0\end{array}\right]$

\section{$7 \quad$ Final comments}

In $[8,9,10]$ the authors introduced and investigated matrices $A$ such that $K A K=A^{s+1}$ for a fixed $K$ involutory and a natural $s \geq 1$. In those papers, it was proved that those matrices are always diagonalizable and a deep spectral theory was developed for them as well as the relation with several wellknown classes of matrices. In addition, some algorithms were given to construct those matrices.

Lastly, in [11], the case for nonsingular matrices was analyzed. Some applications were given for the study of blurring/deblurring images where nonsingularity is crucial.

It is clear that a combination of both situations may be developed in a very similar way. That is, we can define matrices $A$ such that $K A K=A^{-(s+1)}$ for a fixed $K$ involutory and a natural $s \geq 1$. In this manner, all integer powers will be covered. The class of these matrices will be called $\mathbf{G C I}_{K,-s}$.

It can be easily demonstrated that $\mathbf{G C I}_{K,-s}$ is a subclass of nonsingular matrices whose determinant is a root of unity of order $s+2$.

In order to characterize matrices in $\mathbf{G C I}_{K,-s}$, first of all it is observed that $K A K=A^{-(s+1)}$ is equivalent to $A=A^{(s+1)^{2}}$. Thus, the eigenvalues are roots of unity of order $(s+1)^{2}-1$; the set of all of these roots is denoted by $\Omega_{(s+1)^{2}-1}$ where $\omega_{s}$ is a primitive one. 
Then, it should be stated a bijection as in [8]. In fact, let $\psi:\{1,2, \ldots,(s+$ $\left.1)^{2}-2\right\} \rightarrow\left\{1,2, \ldots,(s+1)^{2}-2\right\}$ be defined by $\psi(j)=b_{j}$ where $b_{j}$ is the smallest nonnegative integer such that $b_{j} \equiv-j(s+1)\left[\bmod \left((s+1)^{2}-1\right)\right]$. This function is a bijection.

Now, we establish the characterization.

Theorem 23 Let $A \in \mathbb{C}^{n \times n}$ and $s$ be a positive integer number. Then, the following conditions are equivalent:

(a) $A \in \mathbf{G C I}_{K,-s}$.

(b) $A$ is diagonalizable, $\sigma(A) \subseteq \Omega_{(s+1)^{2}-1}, K P_{j} K=P_{\psi(j)}$ for $j \in\{1,2, \ldots,(s+$ $\left.1)^{2}-2\right\}$ and $K P_{(s+1)^{2}-1} K=P_{(s+1)^{2}-1}$ with $\psi$ being the bijection previously defined and $P_{1}, P_{2}, \ldots, P_{(s+1)^{2}-1}$ are the projectors of the spectral decomposition associated to the eigenvalues $\omega_{s}, \omega_{s}^{2}, \ldots, \omega_{s}^{(s+1)^{2}-2}, 1$, respectively.

(c) $A^{(s+1)^{2}}=A, K P_{j} K=P_{\psi(j)}$ for $j \in\left\{1,2, \ldots,(s+1)^{2}-2\right\}$ and $K P_{(s+1)^{2}-1} K=$ $P_{(s+1)^{2}-1}$ with $\psi$ being the bijection previously defined and $P_{1}, P_{2}, \ldots, P_{(s+1)^{2}-1}$ are the projectors of the spectral decomposition associated to the eigenvalues $\omega_{s}, \omega_{s}^{2}, \ldots, \omega_{s}^{(s+1)^{2}-2}, 1$, respectively.

Curiously, matrices in $\mathbf{G C I}_{K,-s}$ are back within the set of diagonalizable ones.

Although they are not included in this paper, following the same lineaments as in $[8,9,10]$, we can reproduce most of the results given there.

\section{Acknowledgements}

This paper was partially supported by Ministerio de Economía y Competitividad of Spain (Grant number DGI MTM2013-43678-P and Grant Red de Excelencia MTM2017-90682-REDT).

\section{References}

[1] I. Abu-Jeib, Centrosymmetric matrices: Properties and an alternative approach, Canadian Applied Mathematics Quarterly, 10, 4, 429-445, 2002.

[2] Z. Bai, The inverse eigenproblem of centrosymmetric matrices with a submatrix constraint and its approximation, SIAM Journal on Matrix Analysis and Applications, 26, 4, 1100-1114, 2005.

[3] A. Ben-Israel, T. Greville, Generalized Inverses: Theory and Applications, John Wiley \& Sons, Second Edition, 2003.

[4] A. Cantoni, P. Butler, Eigenvalues and Eigenvectors of Symmetric Centrosymmetrlc Matrices, Linear Algebra and its Applications, 13, 275-288, 1976.

[5] M. El-Mikkawy, F. Atlan, On Solving Centrosymmetric Linear Systems, Applied Mathematics, 4, 21-32, 2013. 
[6] P. Gaudreau, H. Safouhi, Centrosymmetric Matrices in the Sinc Collocation Method for Sturm-Liouville Problems, EPJ Web of Conferences, 108, 01004, 2016, DOI: $10.1051 /$ epjconf/201610801004.

[7] N. Higham, Function of matrices: Theory and Computation, SIAM, 2008.

[8] L. Lebtahi, O. Romero, N. Thome, Characterizations of $\{K, s+1\}$-Potent Matrices and Applications, Linear Algebra and its Applications, 436, 293-306, 2012.

[9] L. Lebtahi, O. Romero, N. Thome, Relations between $\{K, s+1\}$-Potent Matrices and Different Classes of Complex Matrices, Linear Algebra and its Applications, 438, 1517-1531, 2013.

[10] L. Lebtahi, O. Romero, N. Thome, Algorithms for $\{K, s+1\}$-potent matrix constructions, Journal of Computational and Applied Mathematics, 249, 157$162,2013$.

[11] L. Lebtahi, O. Romero, N. Thome, Generalized centro-invertible matrices with applications, Applied Mathematics Letters, 38, 106-109, 2014.

[12] A. Lee, Centrohermitian and Skew-Centrohermitian Matrices, Linear Algebra and its Applications, 29, 205-210, 1980.

[13] J. Stuart, J. Weaver. Matrices that commute with a permutation. Linear Algebra and its Applications, 150, 255-265, 1991.

[14] J. Weaver. Centrosymmetric (cross-symmetric) matrices, their basic properties, eigenvalues, and eigenvectors, American Mathematical Monthly, 92, 711-717, 1985.

[15] R.S. Wikramaratna, The centro-invertible matrix: a new type of matrix arising in pseudo-randon number generation, Linear Algebra and its Applications, 434 (1) 144-151, 2011.

[16] R.S. Wikramaratna, The additive congruential random number generator-A special case of a multiple recursive generator, Journal of Computational and Applied Mathematics 216, 2, 371-387, 2008.

[17] M. Yasuda, Some properties of commuting and anti-commuting $m$-involutions, Acta Mathematica Scientia, 32 (2), 631-644, 2012.

[18] L. Zhongyun, Some properties of centrosymmetric matrices and its applications. Numerical Mathematics, 14, 2, 2005. 